\title{
New 63U ATCA rack: thermal performances and integration challenge
}

\author{
Michal Kalinowski ${ }^{1}$ \\ Cracow University of Technology, Mechanical Department \\ al. Jana Pawla II 37, 31-864 Cracow, Poland \\ E-mail: michal.kalinowskiecern.ch
}

\section{Claudio Bortolin}

CERN European Laboratory for Particle Physics

Geneva CH-1211, Switzerland

E-mail: claudio.bortolinecern.ch

To save the space in the underground counting rooms during the ATLAS phase II upgrades, a project dedicated to the study of the impact of installing taller racks within the counting rooms was launched, analysing its cooling performance and the impact on the cooling infrastructures.

A new 63U prototype rack equipped with three ATCA shelves, with open-bottom to top airflow, high-power dissipating load blades, three new prototype $2 \mathrm{U}$ heat exchangers and various sensors was installed in a lab. The impact on the cooling performance of stacking three ATCA crates within a rack was checked, along with other critical aspects.

\section{${ }^{1}$ Speaker}




\section{Introduction}

To meet the DAQ and Trigger requirements, in terms of new ATCA based racks [1], which are to be installed in the underground counting rooms for the ATLAS phase II upgrades, a project dedicated to study the impact of taller rack integration was launched, analysing its cooling performance and the impact of its integration on the general infrastructure. The most valuable aspect in the underground area is space, which due to the nature of the location is limited and cannot be expanded. Thus, an investigation of integrating taller racks, able to house more equipment, into the underground counting rooms was required. In order to do that, a $63 \mathrm{U}$ prototype rack was ordered and installed in a lab. It was equipped with three ATCA shelves. A shelf can house up to 14 blades. The target max power dissipation foreseen is $450 \mathrm{~W}$ per blade, thus the total power dissipated by the rack, including the required internal fan power, can easily exceed $20 \mathrm{~kW}$. This high-power dissipation, called for an assessment of the new taller rack, equipped with three open-bottom to top airflow ATCA crates. To be able to provide the rack with enough cooling power, a chiller with maximum cooling capacity of $25 \mathrm{~kW}$ was ordered and installed - supplying the rack with cooled water. Additionally, new prototype $2 \mathrm{U}$ heat exchangers, optimised for ATCA, were ordered and installed. These require half the number of water connections compared to the previous layout that used 2x1U heat exchangers stacked above each other, simplifying the installation and reducing the risk of a leak. The first 4 racks will be installed in the underground counting rooms during the long shutdown 2 (LS2) in 2019. As previously measured, a standard 52U LHC rack with two ATCA shelves installed, can create noise up to 102 $\mathrm{dB}(\mathrm{A})$, equipping the rack with additional shelf will only increase the generated noise. Therefore, a significant part of this project was dedicated to the study of soundproofing the $63 \mathrm{U}$ rack, and its impact on the cooling performance [2].

\section{Test rack setup}

The new 63U prototype rack, was equipped with:

- Standard LHC rack vertical cooling tower - turbine unit, side panels and air deflector

- Three ATCA shelves with open-bottom to top airflow

- Three newly designed $2 U$ heat exchangers

- Two 3U Power supply units, each capable of providing up to $14.5 \mathrm{~kW}$ of electrical power

- Soundproofing material installed on the front-side panels, rear and front door

- 5 air velocity sensors - UAS1000 [3]

- 20 temperature sensors - PT100 [4]

\subsection{New heat exchangers}

Heat exchangers which are currently used in the Counting room racks, are not optimized to work with ATCA technology. The difference in depth of this equipment, is causing $34 \%$ of the heat exchange area to be unused. Therefore, new prototype $2 \mathrm{U}$ heat exchangers were ordered and tested. 


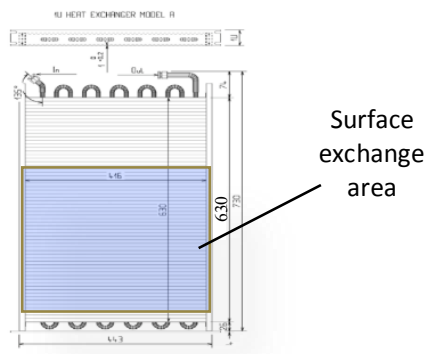

$1 \mathrm{UX}$

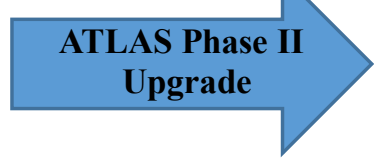

Fig. 1: $2 U$ and $1 U$ heat exchangers.

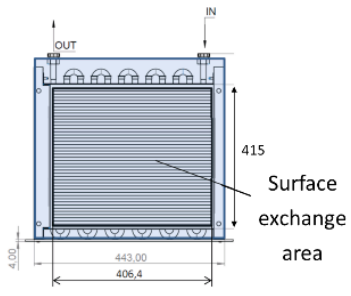

$2 \mathrm{U} \mathrm{HX}$

Both types of heat exchangers have similar performances in terms of cooling. The power removed from the rack in both cases is around $82 \%$ of the total power delivered. Nevertheless, using the new type of HX reduces the number of necessary water connections in the rack by half, as well as preventing installation of Plexiglas panels over non-used heat exchange area. This is why the first four $63 \mathrm{U}$ racks, which are to be installed in the ATLAS underground counting rooms at the beginning of LS2 on January 2019, will be equipped with this new type heat exchangers.

\section{Integration challenge}

Another matter that needs to be considered is that all of the infrastructure in the Counting rooms are designed for $52 \mathrm{U}$ racks, which means that they need to be modified in order to install the taller ones. Due to the unusual dimensions and weight of the new $63 \mathrm{U}$ racks their integration into the Counting rooms can be problematic. There are several aspects which have to be taken under consideration. The first one to consider, is the height of the rack $(300 \mathrm{~cm})$ which is only 44 $\mathrm{cm}$ less than the ceiling height in the ATLAS counting room level 2, additionally due to the large weight of the racks (approx. $500 \mathrm{~kg}$ ), their distribution on the support frame must be carefully planned.

\subsection{Soundproofing}

Since the ATCA fans working at maximum speed generate noise exceeding $100 \mathrm{~dB}(\mathrm{~A})$, a soundproofing material had to be installed on the level of the rack. Collaboration with a company specialising in the field of soundproofing took place during the project. The company suggested two types of soundproofing material:

- Two layer soundproofing using a very dense polymer mass (3.6 mm thick) and non-flammable polyutherane foam ( $25 \mathrm{~mm}$ thick)

- Soundproofing material based on the principle of Helmholtz resonator [7]

During the test campaign, only the first option was tested, the second option is to be evaluated as a part of the collaboration between CERN and the soundproofing company. Taking under consideration that the tested option is also installed in the air corridors of the rack reducing their depth by $28 \mathrm{~mm}$ out of $65 \mathrm{~mm}$, its impact on cooling performance had to be checked. As can be seen on the next page, the results show that the installation of the soundproofing material increases the average maximum temperature by $2.6^{\circ}$, while lowering the noise level by around $3.7 \mathrm{~dB}(\mathrm{~A})$. Since the noise generated by the racks is enormous and soundproofing only on the level of the rack is not enough, installation of soundproofing walls, insulating the area with the ATCA racks in the underground counting rooms is considered. These studies are undergoing and new solutions will be tested soon. 


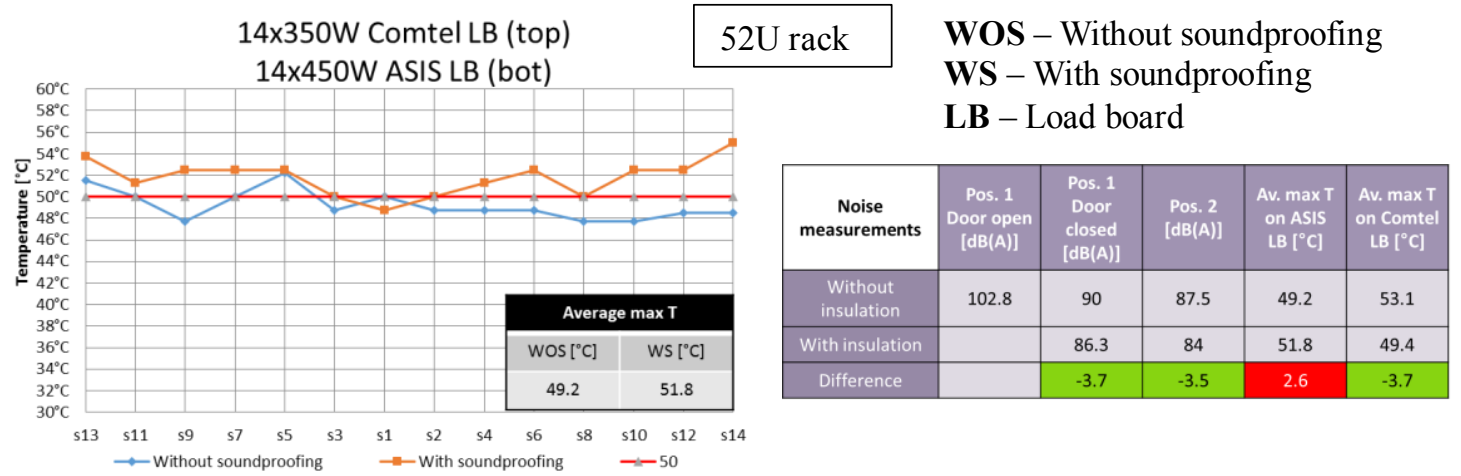

Fig. 2: Soundproofing material installation impact on the cooling performance (left). Noise reduction measurements (right). Presented results come from 52U rack [2].

\section{Thermal tests}

As the cooling performance of the rack equipped with 3 ATCA shelves needed to be evaluated, a series of tests were carried out. The results are shown in the following sections.

\section{1 - $52 \mathrm{U}$ vs $63 \mathrm{U}$}

To determine which of the racks is more efficient in terms of cooling, the following tests were carried out:

- $\quad 52 \mathrm{U}$ (not soundproofed): ATCA 1 (bottom) - 14x400 W, on ASIS LB

ATCA 2 (top) - 14x350 W, on old type Comtel LB

- 63U (soundproofed): ATCA 1 (bottom) - 14x400 W, on ASIS LB

ATCA 2 (middle) - 14x $400 \mathrm{~W}$, on new type Comtel LB

ATCA 3 (top) - 14x350 W, on old type Comtel LB

As the results show, the difference in the cooling performance is significant - the delta between average maximum temperatures of the two racks is $5^{\circ}$. Nevertheless, taking under consideration the extra amount of power delivered to the rack the cooling efficiency is acceptable.

$52 U$ vs $63 U$ - cooling comparison $14 \times 400$ W ASIS LB

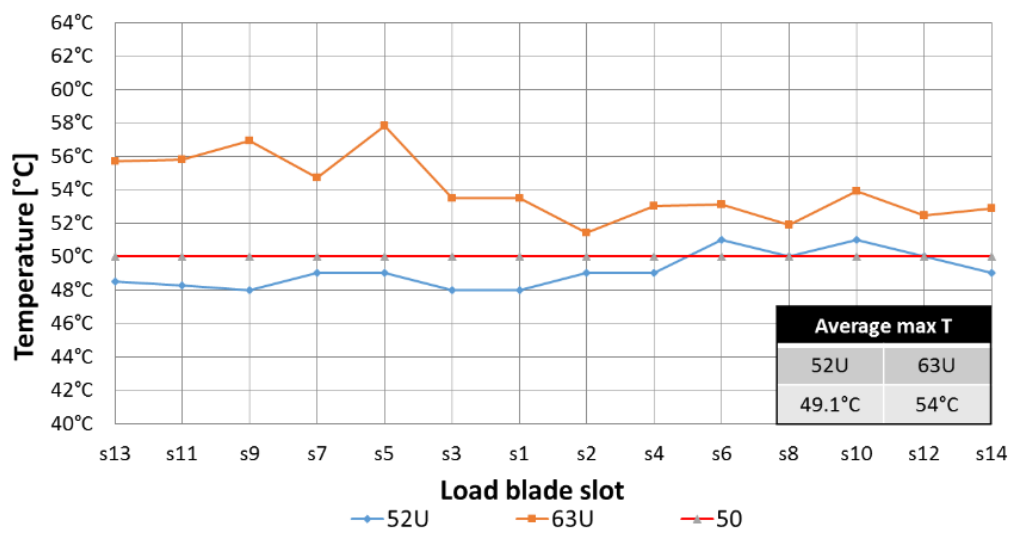

Fig. 3: $52 U$ vs $63 U$ cooling performance. 


\subsection{Cooling water influence}

The flow and temperature of the cooling water have impact on the cooling performance of the rack. Currently maximum water flow available in the counting rooms is $1.8 \mathrm{~m} 3 / \mathrm{h}$, with $15^{\circ} \mathrm{C}$ inlet water temperature. If these parameters could be modified - i.e. decreasing flow in lower powered racks, to increase flow in the one with higher load, the temperature could be reduced from $57.2{ }^{\circ} \mathrm{C}$ to $53.1{ }^{\circ} \mathrm{C}$, which brings the system closer to the $50{ }^{\circ} \mathrm{C}$ requirement.

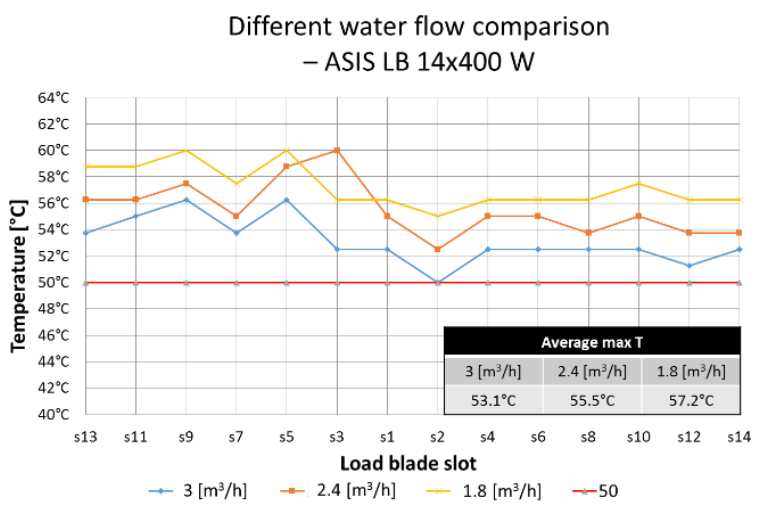

Fig. 4: Different water flow comparison

\section{Conclusions}

The installation of the $63 \mathrm{U}$ racks in the underground counting rooms will be a challenge; the tests carried out so far have demonstrated that the thermal performance of the new $2 \mathrm{U}$ heat exchangers is sufficient and the thermal management is still in line with the 52U racks tests [2]. Additional tests to optimize the fluid distribution to get a more homogenous temperature distribution in the shelf volume will be carried out soon. Tests with real boards will be necessary in order to identify effects of different air flow in different slots. Solutions have also been found for transporting the racks into the counting room and installing them, as well as the installation of larger cooling pipes. The soundproofing of racks and the possibility of building some noise confinement walls are still options under study in close collaboration with a specialized company.

\section{References}

[1] M. Di Cosmo et al., "Evaluation results of xTCA equipment for HEP experiments at CERN" TWEPP 2013 Perugia (Italy)

[2] C. Bortolin, "ATCA thermal management study for the ATLAS phase II upgrades" - TWEPP 2017 Santa Cruz (USA)

[3] https://www.degreec.com/en/airflow-sensing-products/development-use/usb-sensors/uas.html

[4] http://www.itsirl.com/pt100theory.php

[5] http://www.asis-pro.com/atca-systems/600W-Load-Blade

[6] COMTEL ATCA Load Boards, http://www.comtel-online.com/index.php/products/atca/atca-testblades

[7] https://newt.phys.unsw.edu.au/jw/Helmholtz.html 